\title{
Isotopic production cross sections and kinematics of spallation residues from the reaction ${ }^{238} \mathrm{U}(1 \mathrm{AGeV})+\mathrm{d}$
}

\author{
E. Casarejos ${ }^{1, \text { a }}$, J. Pereira ${ }^{1}$, J. Benlliure ${ }^{1}$, P. Armbruster ${ }^{2}$, M. Bernas ${ }^{3}$, A. Boudard ${ }^{4}$, T. Enqvist ${ }^{2}$, S. Leray ${ }^{4}$, B. Mustapha ${ }^{3}$, \\ F. Rejmund ${ }^{3}$, K.-H. Schmidt ${ }^{2}$, C. Stéphan ${ }^{3}$, J. Taieb ${ }^{2}$, L. Tassan-Got ${ }^{3}$, C. Volant ${ }^{4}$, and W. Wlazlo ${ }^{4}$ \\ 1 Universidade de Santiago de Compostela, 15782 Santiago de Compostela, Spain \\ 2 Gesellschaft für Schwerionenforschung, 64291 Darmstadt, Germany \\ 3 Institut de Physique Nucléaire, 91406 Orsay, France \\ 4 DAPNIA-SPhN-CEA, 91191 Saclay, France
}

\begin{abstract}
Isotopic production cross sections and momentum distributions of more than 1400 residual nuclei produced in the collision of ${ }^{238} \mathrm{U}(1 \mathrm{AGeV})+{ }^{2} \mathrm{H}$ have been measured. The experiment was performed at GSI where we could take profit of the inverse kinematics technique and the high-resolution magnetic spectrometer FRS to identify in atomic and mass numbers all the produced nuclei. The identification technique challenges for the case of ${ }^{238} \mathrm{U}$ are reviewed. Some features of ${ }^{238} \mathrm{U}$ residues are discussed, including the access to information of fission dynamics.
\end{abstract}

\section{Introduction}

A large experimental program was initiated at GSI in 1996 to determine the production cross sections of the residues in relativistic heavy-ion fragmentation collisions [1]. Grazing impacts of nuclei/nucleons into a heavier target nuclei induce a pre-equilibrium emission, and the formation of an excited prefragment. Its de-excitation either by particle/cluster emission or fission defines the reaction mechanism leading to the residue. The GSI project, supported by other European institutions, has largely succeeded in providing relevant nuclear data for accelerator-driven systems ADS [2], and the production of intense radioactive ion beams RIBs [2].

The isotopic distributions of fragmentation residues of the reactions induced by $2 \mathrm{GeV}$ deuterons in ${ }^{238} \mathrm{U}$ were measured $[3,4]$, see figure 1 , and some results are discussed in this work. The experiment was performed in inverse kinematics at GSI. The primary reaction products were fully identified in mass and atomic number prior to beta decay, using the fragment separator FRS. This technique allows an unambiguous identification of the residues, and gives access directly to the primary reaction production, thus allowing the study of the reaction mechanisms.

Different Monte Carlo simulation codes pursue in the description of these processes and the evaluation of the cross sections. Despite their capacity to describe the general trends of the measured data, these codes presented a poor predictive power few years ago [5]. The large benchmark data collection provided by our collaboration has made it possible a deep study of the spallation reaction mechanisms. An increasing satisfactory description of the data by some codes [6] resumes the progresses in the understanding of spallation reactions, and the key roll of high-quality data in this progress.

\section{Experimental technique}

The experiment was performed at GSI by shooting a ${ }^{238} \mathrm{U}$ beam accelerated in the SIS synchrotron up to $1 \mathrm{AGeV}$, into

\footnotetext{
${ }^{a}$ Presenting author, e-mail: casarejo@usc.es
}

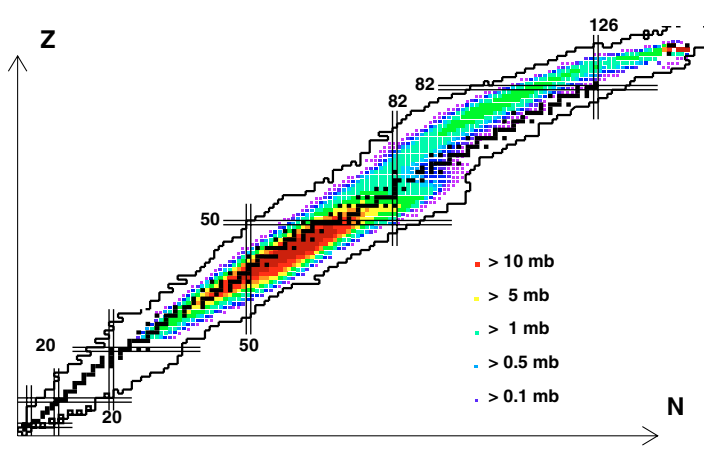

Fig. 1. Two-dimensional cluster plot of all nuclei produced as projectile residues with a cross section larger than $100 \mu \mathrm{b}$ in the reaction ${ }^{238} \mathrm{U}(1 \mathrm{AGeV})+{ }^{2} \mathrm{H}$ presented on top of a chart of the nuclides $[3,4]$. The (colors) sizes represent the production cross section, the black squares correspond to stable isotopes, and the lines indicate the limit of the known nuclides.

a cryogenic liquid-deuterium target of $200 \mathrm{mg} / \mathrm{cm}^{2}$. The beam intensity, up to $10^{7}$ particles/s, was measured continuously by a well suited monitor. The residual nuclides were identified both in mass and atomic numbers, while flying forward, using the FRagment Separator FRS [7], and a dedicated detection setup. The FRS machine, a 70 meters long, zero-degree magnetic spectrometer, allowed the separation of the heavy residues with good resolution $(\mathrm{A} / \Delta \mathrm{A} \sim 400$ for $\mathrm{A} \sim 238)$ with a very exigent technique. The short times of flight involved $(<200 \mathrm{~ns})$ allowed to observe the primary production of the reaction residues, overcoming the radioactive decay drawback present in spallation/direct-kinematic experiments [8].

The measurement of the magnetic fields of the FRS and the position of the particles in the magnetic dispersion coordinate at the focal planes of the FRS, defined the magnetic rigidity $B \rho=A u c \beta \gamma / Q e$ of each residue. The time of flight between two focal planes of the FRS $(35 \mathrm{~m})$ defined the relativistic $\beta \gamma$ value; therefore the ratio of mass number A to ionic charge $\mathrm{Q}$ was obtained. The proper assignation of ionic charge $\mathrm{Q}$ and atomic number $\mathrm{Z}$ is a challenge for nuclides with $\mathrm{Z}$ 


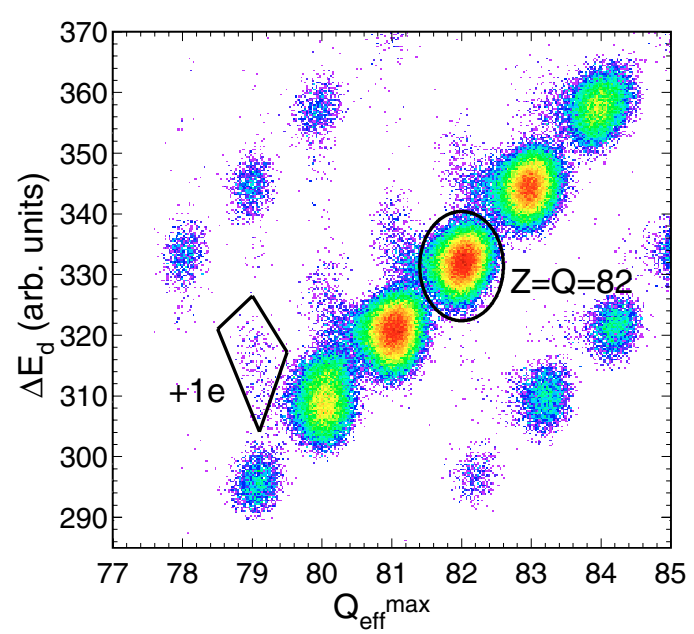

Fig. 2. Scatter plot of the energy loss in the degrader $\Delta \mathrm{E}_{d}$ as a function of charge Q, measured with the two ionisation chambers. The FRS magnetic setting was centred around ${ }_{82}^{195} \mathrm{~Pb}$. The bare nuclei, along the FRS, of element $Z=82$ are indicated. See ref. [3] for details.

above 70 at these energies, since the charge state of a nucleus after traversing any layer of matter may change. We used ionisation chambers to measure the energy loss of the ions, thus Q. However, this does not provide Z straightforward. The limited resolution for the higher charges is also a major problem. Additionally one we must know the charge $\mathrm{Q}$ within the spectrometer to define correctly A from the measured $B \rho$ value. To deal with this situation we have developed an outstanding method that allow us to obtain both $\mathrm{Z}$ and $\mathrm{Q}$ with high resolution, along the FRS. The measurements of the energy losses in two ionisation chambers were combined to improve the charge resolution. A $\mathrm{Nb}$ stripping foil placed in between both chambers enhanced the separation of charge states, thus Z. Our detectors also allowed to define the energy loss induced by a profiled achromatic aluminum degrader $\left(\sim 4 \mathrm{~g} / \mathrm{cm}^{2}\right)$ in the intermediate focal plane of the FRS, providing alternatively the definition of $\mathrm{Q}$. The change in magnetic rigidity before and after the degrader, gives another measurement of Q. Two Nb stripping foils installed after the target and the degrader, enhanced the fraction of bare ions along the spectrometer. The comparison of the measurements obtained from the ionisation chambers and the $B \rho$ shift due to the degrader, as shown in figure 2, defines the charge $\mathrm{Q}$ of the nuclides at different positions of the FRS. With this method, detailed in ref. [3], the selection in $\mathrm{Z}$ and $\mathrm{Q}$ is unambiguous, $\mathrm{A}$ is correctly defined, and the isotopic separation resolution largely improved. The use of an energy degrader becomes a key piece when working with heavy nuclides. Also the use of the detector setup to their maximal resolutions, allowed us to succeed in our purpose.

For residues with $\mathrm{Z}$ below 70 , the identification needs no more the degrader setup. Softer conditions and the resolution of the FRS allow easily to access the identification.

\section{Cross sections}

To define the production cross sections of the reaction residues, we had to correct the measured counting rates of each identified nuclide for the different effects inherent to our experimental method, previous to the normalization to the target thickness and beam intensity. The factors that correct the yields are briefly mentioned here, and extensively described in ref. [3]. The dead time of the whole acquisition system, typically below $20 \%$, was monitored with an accuracy within $1 \%$. The uncertainty in beam intensity was $4 \%$ after the calibration of the beam monitor. The uncertainty in the target thickness was calculated including the deformation of the target walls, beam alignment and beam profile, resulting in $3 \%$. The statistical accuracy was kept well below $5 \%$ for most of the nuclides. The statistical quality of the data is shown in the smoothness of the isotopic cross sections (see, e.g., $[3,4]$ ).

The limited longitudinal momentum acceptance of the spectrometer was overcome by overlapping several magnetic settings of the FRS, scanning the whole longitudinal momentum distribution for each nucleus. The angular distributions, of few mrad, were fully accepted for evaporation residues (with transmissions close to 100\%). In the case of fission residues the transmission correction can be large; however our corrections always kept low uncertainties, see ref. [4].

We measured the momentum distributions, and thus the yields, of all the bare nuclides produced. The calculations to correct for the rest of charge states were cross checked with values corresponding to different charge states we obtained in out magnetic settings. The uncertainty of this correction was established in 5\%. The secondary reactions in any layer of matter along the path of the detection, were also corrected. The losses in the degrader amount up to $\sim 40 \%$ for the heavier residues. The cross section values for these processes were calculated with an accuracy of $10 \%$, cross checked with the losses we measured for the beam. The contributions from the target container, of few percent, where subtracted, inducing a rather low uncertainty in the yield values.

We note that the yields have been measured as function of the (longitudinal) momentum, thus having access to the momentum distributions, which have been also determined. The characteristics of the kinematics after evaporation and fission, as well as the FRS transmission influence, were used to disentangle the reaction contributions of a part of the observed nuclei, produced by the two reaction mechanisms, particle emission and fission.

\section{Results and discussion}

In this work we have measured more than 1400 isotopic cross sections of projectile residues produced in the reaction ${ }^{238} \mathrm{U}(1 \mathrm{AGeV})+{ }^{2} \mathrm{H}$, with typical uncertainties between $10 \%$ and $20 \%$. From the excited projectile pre-fragments produced in the interaction between the projectile and the target nuclei, the production of any residue is determined by the competition among the open de-excitation channels: particle emission (mostly neutrons and protons) and fission. Considering the excitation energies involved in this reaction, we could eventually observe the onset of multi-fragmentation. However such discussion is well beyond the scope of this paper.

In figure 1 we represent in a cluster plot the measured cross sections on top of a chart of the nuclides. The upper part of the chart of the nuclides is mostly populated by 
neutron-deficient nuclides covering a large range in charge from the projectile nucleus down to the charge 65. The isotopic distributions cover typically around 20 nuclides with a maximum production around a few milibarns. These nuclei are produced in a nucleon and/or cluster emission process from the excited projectile pre-fragments. This process leads to residual nuclei with an isotopic composition determined by the competition between neutron and proton emission. Since these two emissions differ mainly in the proton Coulomb barrier, the equilibrium between both processes is reached at the left of the stability line, along the so called evaporation corridor. The initial excitation energy $\mathrm{E}^{*}$ determines the length of the evaporation chain and consequently the mass of the final residue. Since the number of nucleons removed from the projectile in direct collisions with the target deuteron is expected to be rather small, the mass of the evaporation residues is an observable strongly correlated with the $\mathrm{E}^{*}$ induced in the reaction.

The second group of nuclei corresponds to medium-mass fragments with atomic numbers between 23 and 65. The isotopic distributions are broader, populating on average 25 isotopes. The distributions are centred to the right of the stability line, and the production cross sections are much larger than the ones observed for the evaporation residues. These nuclei are expected to be produced by fission of the projectile excited pre-fragments.

The fission process preserves the neutron excess of the fissioning nucleus at low $\mathrm{E}^{*}$. However, when the system crosses the fission saddle at high $\mathrm{E}^{*}$, this neutron excess is partially lost by pre- and post-saddle neutron evaporation, both from the system on the way to scission and from the fragments, shifting and broadening the final isotopic distribution of fission residues. The observed mass distribution of fission residues is mostly single-humped, in contrast to the well known doublehumped distribution of residues produced in the low-energy fission of ${ }^{238} \mathrm{U}$, indicating in our case that fission takes place at high $\mathrm{E}^{*}$, where shell effects are washed out. Nevertheless, the most neutron-rich fission residues show a contribution by a double-humped component resulting from low-energy fission induced in very peripheral reactions.

The momenta of the evaporation residues contain very valuable information for technical applications, mainly connected with the material damages in ADS. In the case of fission residues the momenta allow to obtain the total kinetic energy, giving access to the definition of the fissioning nuclei distribution (in mass and charge) of the residues. Those observations seed valuable information, e.g., about pre- and post-scission emission multiplicities.

\subsection{Comparison to other data}

The data provided by our collaboration made it possible both qualitative and quantitative comparisons. These studies seed light into aspects that definitely improve our understanding of the reaction mechanisms involved.

We can reconstruct the residual production as a function of mass and atomic numbers, since the have access directly to the major cross sections of the isotopic chains. In figure 3 we show the mass distributions of residues of ${ }^{238} \mathrm{U}(1 \mathrm{AGeV})$ induced by ${ }^{2} \mathrm{H}[3,4]$, and $\mathrm{H}[9]$.

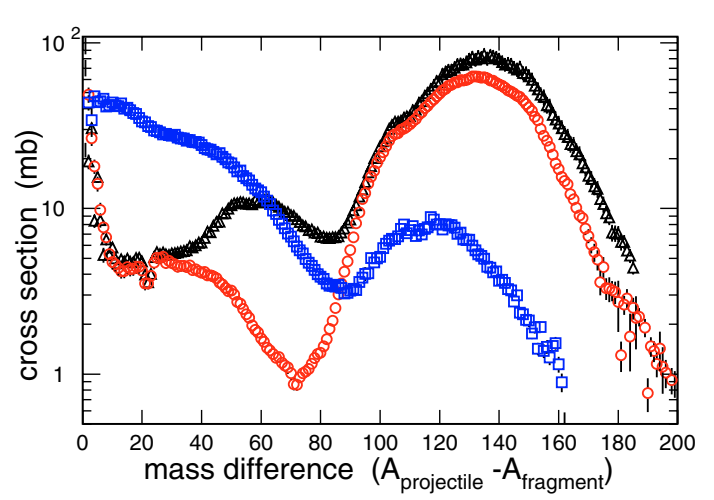

Fig. 3. Mass distributions of the residues in the reactions ${ }^{208} \mathrm{~Pb}(1 \mathrm{AGeV})+{ }^{2} \mathrm{H}$ (squares) [10], ${ }^{238} \mathrm{U}(1 \mathrm{AGeV})+\mathrm{p}$ (circles) [9] and ${ }^{238} \mathrm{U}(1 \mathrm{AGeV})+{ }^{2} \mathrm{H}$ (triangles) $[3,4]$, represented as a function of the difference mass of residue and projectile.

We clearly distinguish the productions connected to evaporation (closer to the projectile mass), and fission processes, as well as a mass range where both processes contribute. The production of the heavier residues from $\mathrm{H}$ and ${ }^{2} \mathrm{H}$ induced reactions almost coincide $(\triangle \mathrm{A}<30)$. The effect is even more remarkable when looking to the isotopic distributions of elements close to the projectile [3]. This observation can be explained if we consider that due to the large spatial distribution of the deuteron, many of the peripheral collisions involve a single nucleon. Therefore both reactions result in the same production. The different $\mathrm{E}^{*}$ distributions induced by $\mathrm{H}$ and ${ }^{2} \mathrm{H}$ determines the different length of the evaporation ranges observed.

In figure 3 we also compare the production of ${ }^{208} \mathrm{~Pb}(1 \mathrm{AGeV})+{ }^{2} \mathrm{H}[10]$. The large differences in the production of heavy residues are due to the fission channel, which largely dominates the reaction mechanism in the neighborhood of the ${ }^{238} \mathrm{U}$, depopulating the production of residues close to the projectile and populating medium-mass residues. The production of neutron-deficient isotopes suffer largely the depletion caused by fission due to the fast evolution of the fission barriers. Being fragmentation reactions the most probable tool for the production of those nuclei, involved, e.g., in the quest for proton-decay candidates, we note that reaching the proton drip-line above $\mathrm{Pb}$ is at the price of rather low cross sections. A similar situation happens for neutronrich isotopes. Fission can populate medium mass regions, see figure 1. However, heavy neutron-rich areas are accessible only by the evaporation channels of the fragmentation reactions. Actually the lowest $\mathrm{E}^{*}$ allows the access to the most exotic regions. The information extracted from our data sets are helping to improve the codes able to provide realistic evaluations of the RIB intensities available in nowadays and future facilities [11].

\subsection{Transient and quasi-stationary dissipative effects}

The comparison of the measured cross sections with Monte Carlo de-excitation codes including an analytical description of the dynamics of fission has shown the sensitivity of the ${ }^{238} \mathrm{U}(1 \mathrm{AGeV})+{ }^{2} \mathrm{H}$ data to nuclear dissipation [12]. 
Key nuclear dynamical results have been recently obtained [13] and implemented in the ABLA code [14].

The advantage of looking to fragmentation reaction mechanisms is that the excited fissioning nucleus is produced with well defined initial conditions: with almost non-deformed nuclei, covering a large range in $\mathrm{E}^{*}$, and introducing only moderate angular moment. These conditions make these data the best suitable data for dynamical model comparison.

The dominant fission channel in this reaction can be investigated from the survival probability against fission. The advantage of looking to the evaporation residues, is that being a large fraction of fission produced by lower $\mathrm{E}^{*}(<200 \mathrm{MeV})$, and the different descriptions of fission dynamics being more important at higher $\mathrm{E}^{*}$, they provide quite similar results for the mass distributions. It also offers the advantage to use the evaporation nuclei for investigating the dependence on $\mathrm{E}^{*}$ of the fission process, because of the strong correlation of $\mathrm{E}^{*}$ and mass.

The fact that for large mass losses the production of residues from both reactions ${ }^{208} \mathrm{~Pb}(1 \mathrm{AGeV})+{ }^{2} \mathrm{H}$ and ${ }^{238} \mathrm{U}(1 \mathrm{AGeV})+{ }^{2} \mathrm{H}$ have similar cross sections, see figure 3 , is a clear signature of the suppression of fission at high $\mathrm{E}^{*}$. This extreme is confirmed by model calculations: the suppression of the fission channel reproduces the evaporation data corresponding to high $\mathrm{E}^{*}$ for both $\mathrm{Pb}$ and $\mathrm{U}$ residues.

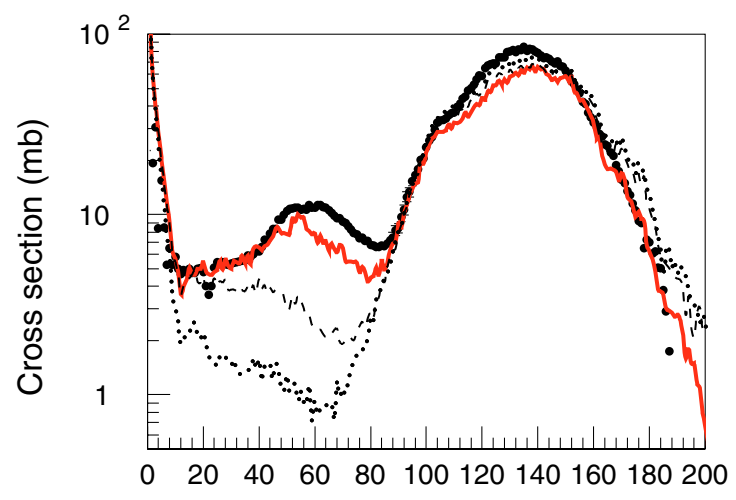

$\Delta \mathrm{A}$

Fig. 4. Measured mass distribution of ${ }^{238} \mathrm{U}(1 \mathrm{AGeV})+{ }^{2} \mathrm{H}$ residues $[3$, 4]. The data (black points) are compared with several model calculations. Dotted line: statistical description of the fission. Dashed line: quasi-stationary effects added by using the Kramer's factor and a reduced fission width $\beta=2 \cdot 10^{21} \mathrm{~s}^{-1}$. Solid line: transient effects added. See ref. [12] for details of the model.

Model calculations also show that the measured data can only be understood when the dynamics of fission is considered. The dotted line in figure 4 was obtained using the statistical model of Bohr and Wheeler. This model clearly overestimates the fission channel depopulating largely the production of evaporation residues. The dashed line corresponds to a calculation where the fission width is evaluated using the so called Kramer's factor, as evaluated in [13] to account for the stationary effects in the fission flux. A calculation considering all the transient effects due to the time dependence of the fission width (solid line) provides the best description of the data. In this case, the time dependence has been calculated according to the approximated solution of the Fokker-Planck equation as proposed in ABLA. The accuracy of the present data, together with the large excitation-energy range covered with this reaction made it possible to characterise and quantify the role of transient and quasi-stationary dissipative effects in the fission flux across the barrier.

\section{Conclusions}

We have measured the production cross sections of more than 1400 residual nuclei produced in the collision of ${ }^{238} \mathrm{U}(1 \mathrm{AGeV})+{ }^{2} \mathrm{H}$, together with their momenta. We deployed an outstanding method for nuclei identification of residues produced in inverse kinematics, allowing to exploit at maximum the resolving power of the FRS. The main features of this reaction were discussed in comparison with other available data sets provided by our collaboration, highlighting the strong influence of the fission channel. These data set a clear benchmark for the studies of the reactions mechanisms of these processes, as showed by the available model that can describe the data.

We are grateful to people who helped in these experiments (S. Czajkowski, R. Legrain, M. Pravikoff); the GSI accelerator and technical staff (K.-H. Berh, C. Burkhard, A. Bruenle); and the CEA cryogenic group (P. Chesny).

\section{References}

1. See the website of the CHARMS collaboration http://wwww2k.gsi.de/charms/.

2. See, e.g., Int. Conf. on Nuclear Data for Science and Technology, Santa Fe, New Mexico, 2004, AIP Conf. Proc. 769.

3. E. Casarejos et al., Phys. Rev. C 74, 044612 (2006).

4. J. Pereira et al., Phys. Rev. C 75, 014602 (2006).

5. M. Blann et al., Int. Code Comparison for Intermediate Nuclear Data, OECD/NEA (1994).

6. A. Boudard et al.(these proceedings).

7. H. Geissel et al., Nucl. Instrum. Meth. B 70, 286 (1992).

8. R. Michel et al., Nucl. Instrum. Meth. B 129, 153 (1997).

9. P. Armbruster et al., Phys. Rev. Lett. 93, 212701 (2004).

10. T. Enqvist et al., Nucl. Phys. A 703, 435 (2002).

11. See the website of EURISOL, http://www.eurisol.org.

12. J. Benlliure et al., Phys. Rev. C 74, 014609 (2006).

13. B. Jurado et al., Nucl. Phys. A 747, 14 (2005), and refs. therein. 14. A. Kelic et al. (these proceedings). 